\title{
Error Propagation and Recovery in Decision-Feedback Equalizers for Nonlinear Channels
}

\author{
John Tsimbinos, Member, IEEE, and Langford B. White, Member, IEEE
}

\begin{abstract}
Nonlinear intersymbol interference is often present in communication and digital storage channels. Decision-feedback equalizers (DFEs) can decrease this nonlinear effect by including appropriate nonlinear feedback filters. Although various applications of these types of equalizers have been published in the literature, the analysis of their stability and error recovery has not appeared. In this letter, we consider a DFE with a nonlinear feedback filter based on a discrete Volterra series. We extend error propagation, error probability, stability, and error recovery time results for $N$ th-order nonlinear channels.
\end{abstract}

Index Terms-Decision-feedback equalizers, error analysis, nonlinear distortion, nonlinear filters, Volterra series.

\section{INTRODUCTION}

$\mathbf{N}$ ONLINEAR intersymbol interference (ISI) caused by channel nonlinearities can significantly increase the error rate in communication and digital storage channels. A decision-feedback equalizer (DFE) is aimed at decreasing the effect of this nonlinear process by incorporating nonlinear feedforward and feedback filters. The nonlinear feedback filter may be of the form of a discrete time Volterra series, a look-up table, or a neural network [1]-[5]. Basic details of DFEs for nonlinear channels, and their applications have been sparsely published in the literature. However, to the best of the authors' knowledge, no work has appeared on the error propagation and recovery of such equalizers. In this letter we consider a DFE with a nonlinear feedback filter based on a discrete $N$ th-order Volterra series, a natural nonlinear extension of the linear finite impulse response (FIR) filter. We determine the effect of the nonlinear extensions on the error propagation analysis, error probability analysis, stability analysis, and error recovery time.

\section{NONLINEAR ChANNEl MODEL}

The discrete time Volterra model [6] is a natural extension of the widely used linear FIR channel model. Such a model has been used to extend the DFE to the nonlinear channel case [4], [5]. The $N$ th-order discrete time Volterra model adopted in this letter is given by (1). For the purpose of our analysis we assume only postcursor terms, and that any precursor terms can be dealt with by prefiltering. $y_{k}$ is the channel output for an

Paper approved by R. A. Kennedy, the Editor for Data Communications, Modulation, and Signal Design of the IEEE Communications Society. Manuscript received November 6, 1999; revised April 15, 2000. This paper was presented in part at the Fifth International Symposium on Signal Processing and its Applications (ISSPA), Brisbane, Australia, August, 1999.

J. Tsimbinos is with the Defence Science and Technology Organization, Salisbury, SA 5108, Australia (e-mail: john.tsimbinos@dsto.defence.gov.au).

L. B. White is with the University of Adelaide, Adelaide, SA 5005, Australia (e-mail:1white@eleceng.adelaide.edu.au).

Publisher Item Identifier S 0090-6778(01)01306-X.

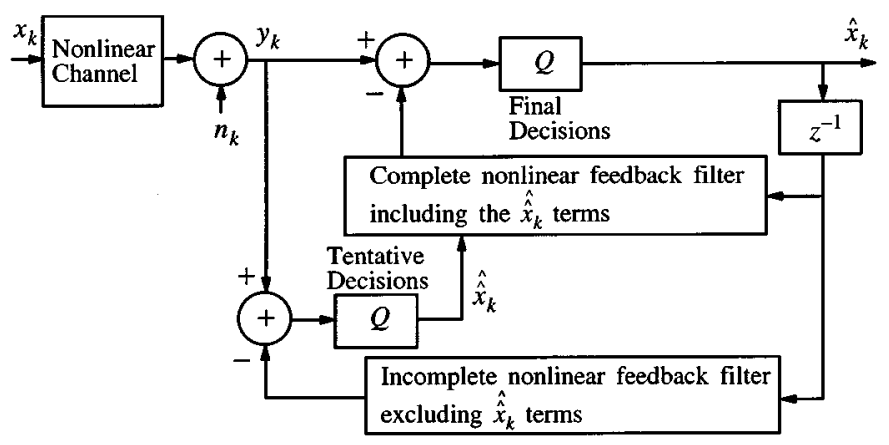

Fig. 1. Nonlinear channel equalizer with Volterra feedback filters.

input $x_{k}$ and white Gaussian noise $n_{k} . h_{i}, h_{i, j}, \ldots, h_{i, j, \ldots, q}$ are the Volterra kernels, and $M_{1}, M_{2}, \ldots, M_{N}$ are the associated memories.

$$
\begin{aligned}
y_{k}= & h_{0} x_{k}+\sum_{i=1}^{M_{1}} h_{i} x_{k-i}+\sum_{i=0}^{M_{2}} \sum_{j=i}^{M_{2}} h_{i, j} x_{k-i} x_{k-j}+\cdots \\
& +\sum_{i=0}^{M_{N}} \sum_{j=i}^{M_{N}} \cdots \sum_{q=p}^{M_{N}} h_{i, j, \ldots, q} x_{k-i} x_{k-j} \cdots x_{k-q}+n_{k} .
\end{aligned}
$$

\section{DFE WITH VOLTERRA FEEDBACK FILTER}

Nonlinear intersymbol interference is a function of previous as well as current samples, necessitating the need for the DFE to make tentative decisions. Such an equalizer is shown in Fig. 1. We separate the nonlinear ISI components not containing the current sample $x_{k}$, and subtract them from the system output $y_{k}$ to obtain a reasonable tentative decision $\hat{\hat{x}}$. For a second-order nonlinear system, a tentative decision would be $\hat{\hat{x}}=Q\left[y_{k}-\right.$ $\left.\sum_{i=1}^{M_{1}} h_{i} x_{k-i}-\sum_{i=1}^{M_{2}} \sum_{j=i}^{M_{2}} h_{i, j} x_{k-i} x_{k-j}\right]$, where $Q[$.] denotes quantization. The final decision of such an equalizer is given by

$$
\begin{gathered}
\hat{x}_{k}=Q\left[y_{k}-\sum_{i=1}^{M_{1}} h_{i} \hat{x}_{k-i}-\hat{\hat{x}}_{k}^{2}-\hat{\hat{x}}_{k} \sum_{j=1}^{M_{2}} h_{0, j} \hat{x}_{k-j}\right. \\
\left.-\sum_{i=1}^{M_{2}} \sum_{j=i}^{M_{2}} h_{i, j} \hat{x}_{k-i} \hat{x}_{k-j}\right] .
\end{gathered}
$$

We define the first-order error variable as $e_{k-i}^{(1)}=$ $\hat{x}_{k-i}-x_{k-i}$, the second-order error variable as $e_{k-i, k-j}^{(2)}=$ $\hat{x}_{k-i} \hat{x}_{k-j}-x_{k-i} x_{k-j}$ for $i=1,2, \ldots, M_{2}, j=$ $i, 2, \ldots, M_{2}$, and $e_{k, k-j}^{(2)}=\hat{\hat{x}}_{k} \hat{x}_{k-j}-x_{k} x_{k-j}$. The 
$N$ th-order error is $e_{k-i, k-j, \ldots, k-q}^{(N)}$, and is similarly defined. The final decision estimate can be written as

$$
\begin{aligned}
\hat{x}_{k}=Q & {\left[h_{0} x_{k}+n_{k}-\sum_{i=1}^{M_{1}} h_{i} e_{k-i}^{(1)}-\sum_{i=0}^{M_{2}} \sum_{j=i}^{M_{2}} h_{i, j} e_{k-i, k-j}^{(2)}\right.} \\
& \left.-\cdots-\sum_{i=0}^{M_{N}} \sum_{j=i}^{M_{N}} \cdots \sum_{q=p}^{M_{N}} h_{i, j, \ldots, q} e_{k-i, k-j, \ldots, k-q}^{(N)}\right] .
\end{aligned}
$$

We consider an $m$-ary random data sequence $\left\{x_{k}\right\}$, independent and identically distributed. The corresponding decision device $Q[$.$] is an m$ level quantizer. We can write

$$
\begin{gathered}
x_{k} \in\{-(m-1),-(m-3), \ldots,-1,1, \ldots, m-3, m-1\} \\
e_{k-i}^{(1)} \in\{-2(m-1), \ldots,-2,2, \ldots, 2(m-1)\} \\
e_{k-i, k-j}^{(2)} \in\left\{-2(m-1)^{2}, \ldots,-2,2, \ldots, 2(m-1)^{2}\right\}
\end{gathered}
$$$$
e_{k-i, k-j, \ldots, k-q}^{(N)} \in\left\{-2(m-1)^{N}, \ldots,-2,2, \ldots, 2(m-1)^{N}\right\}
$$

\section{DEFINITION OF ERROR StATES}

The operation of a DFE may involve primary and secondary errors. A primary error is one due to noise in the channel. The amount of noise required to produce a primary error will be determined by the level of the ISI. The primary error may then lead to a secondary error due to the feedback in the DFE. Error states may be defined according to past and present errors made in the DFE.

We can carry out an error propagation analysis of a DFE for nonlinear channels by considering past error states. We consider the aggregated Markov states model introduced in [7] and used in [8]-[13] for the linear channel case. This model groups the Markov states according to the most recent error, and is used for determining error probability and error recovery times. This model can also be used for the $N$ th-order nonlinear channel case considered in this letter.

A prerequisite for obtaining an $N$ th-order error $e_{k-i, k-j, \ldots, k-q}^{(N)}$ associated with an $N$ th-order product of decisions, is the existence of at least one first-order error $e_{k-i}^{(1)}$, which has a common time instance with the $N$ th-order error. Therefore, the time extent of higher-order errors can be defined in terms of the time extent of first-order errors. If there are no first-order errors up to a particular time instant, we can say that all higher-order errors involving products of decisions up to these time instances are also zero. Therefore, for an $N$ th-order nonlinear channel, the error states $\Phi_{l}$ states can still be defined according to the position of the most recent first-order error $e_{k-l}^{(1)}$, but for a maximum channel memory $M=\max \left\{M_{1}, M_{2}, \ldots, M_{N}\right\}$ that takes into account the memory of the nonlinear components. $\Gamma$ is the zero error state. Table I gives a representation of the states, and the state transition diagram is given in Fig. 2. For the linear channel case where $M=M_{1}$, the steady-state transition probabilities
TABLE I

State Assignment for the $N$ Th-ORder Nonlinear Channel CASE $(\mathrm{E}=\mathrm{ERROR}, 0=$ No ERror, $\mathrm{X}=$ Do NOT CARE $)$

\begin{tabular}{|l|l|}
\hline State & Error-Pattern $\left(e_{k-1}^{(1)}, e_{k-2}^{(1)}, \ldots, e_{k-M}^{(1)}\right)$ \\
\hline$\Phi_{1}$ & $E X X X X \ldots X X X X X$ \\
$\Phi_{2}$ & $0 E X X X \ldots X X X X X$ \\
$\cdot$ & $\cdot$ \\
$\cdot$ & $\cdot$ \\
$\cdot$ &. \\
$\Phi_{M}$ & $00000 \ldots 0000 E$ \\
$\Gamma$ & $00000 \ldots 00000$ \\
\hline
\end{tabular}

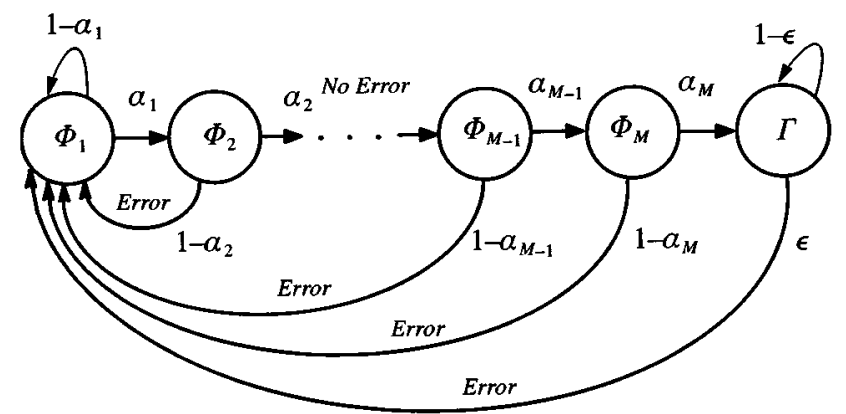

Fig. 2. State transition diagram for $N$ th-order nonlinear system with memory M.

are given in [7]. Similar steady-state transition probabilities can be determined for the $N$ th-order nonlinear channel case by taking into account the full memory of the nonlinear channel: $\alpha_{l}=\lim _{k \rightarrow \infty} \operatorname{Pr}\left\{e_{k}^{(1)}=0 \mid S_{k}=\Phi_{l}\right\}, 1 \leq l \leq M$, where $S_{k}$ is the current state, and $(1 / m) \leq \alpha_{l} \leq 1,1 \leq l \leq M$. $\epsilon$ is the probability of error due to noise in the absence of any past errors.

\section{Stability Analysis}

Stability is defined in terms of the ability for the DFE to recover from the effects of a primary error (return to a zero error state denoted $\Gamma$ ) in a finite error recovery time, in the absence of noise. The sufficient conditions for stability are based on an open eye, and do not allow errors to occur without noise, leading to fast error recovery. For the linear channel case, sufficient conditions based on an open eye are given in [11], as

$$
\sum_{i=1}^{M_{1}}\left|h_{i}\right|<\frac{\left|h_{0}\right|}{2(m-1)}
$$

For a channel with up to $N$ th-order nonlinear ISI, the open eye sufficient condition for stability is given by

$$
\begin{aligned}
2(m-1) & \sum_{i=1}^{M_{1}}\left|h_{i}\right|+2(m-1)^{2} \sum_{i=0}^{M_{2}} \sum_{j=i}^{M_{2}}\left|h_{i, j}\right|+\cdots \\
& +2(m-1)^{N} \sum_{i=0}^{M_{N}} \sum_{j=i}^{M_{N}} \cdots \sum_{q=p}^{M_{N}}\left|h_{i, j}, \ldots, q\right|<\left|h_{0}\right| .
\end{aligned}
$$

The sufficient condition for stability given in this section is quite restrictive and may not always be easily satisfied. Determining less restrictive (necessary) conditions for the nonlinear case is beyond the scope of this letter and although it is a problem for further investigation, it is of little practical interest. 
TABLE II

TABLE OF ERROR PROBABILITY EXPRESSIONS FOR NTH-ORDER NONLINEAR CHANNELS

\begin{tabular}{|l|l|}
\hline Condition & Error Probability Bound \\
\hline $\begin{array}{l}\text { Any channel condition, any } \\
\text { SNR }\end{array}$ & $P_{E}^{U}=\frac{1}{m}$ \\
\hline$\sum_{i=1}^{M_{1}}\left|h_{i}\right|+\sum_{i=0}^{M_{2}} \sum_{j=i}^{M_{2}}\left|h_{i, j}\right|+$ & $P_{E}^{U}=\frac{\epsilon(m)^{M}}{2 \epsilon\left((m)^{M}-1\right)+1}$, \\
$\ldots<\infty$, & $M=\max \left\{M_{1}, M_{2}, \ldots M_{N}\right\}$ \\
$\begin{array}{l}h_{i}=0 \text { for } i>M_{1}, h_{i, j}=0 \text { for } \\
i, j>M_{2}, \text { noise present }\end{array}$ & \\
\hline As above, with high SNR & $P_{E}^{U}=\epsilon(m)^{M}$, \\
& $M=\max \left\{M_{1}, M_{2}, \ldots, M_{N}\right\}$ \\
\hline
\end{tabular}

Violating the sufficient conditions in general would result in an increase in the error probability, leading to longer error recovery times. In the extreme case, it may result in no recovery, although the input sequences that result in such a failure have such a special structure, that their occurrence in a communications system would have a probability of zero [11]. Several such "pathalogical" sequences are considered in [11] and [12]. Of more interest is the error probability and error recover times for the various channel conditions. These are dealt with in the following sections.

\section{ERROR PROBABILITY}

DFE error probability for the linear channel case has been studied in [7]-[10]. The work is based on modeling the error transition problem as an aggregated Markov state model [7], and the theory of success runs covered in [14]. Here we have extended the error probability upper bounds $P_{E}^{U}$ of a DFE to the $N$ th-order nonlinear channel case, by considering the memory of the nonlinear terms. The results are summarized in Table II.

\section{ERROR RECOVERY}

The average error recovery time $T_{\mathrm{ER}}$ is the average time for the DFE to transition from the error state $\Phi_{1}$ where a primary error due to noise has occurred, to the zero error state $\Gamma$, under zero noise conditions. Intuitively, from Fig. 2 , the minimum possible error recovery time is $M$. Error recovery of DFEs has been extensively studied for the linear channel case [11]-[13]. The error recovery expressions given in [11] and [12] are independent of the type of channel, and tend to be very much conservative for most practical channels. Reference [13] provides an easy to follow analysis for the linear channel case by expressing the average error recovery time in terms of the transition probabilities $\alpha_{l}$, and then determining bounds for these transition probabilities, which are the same as in [11]. Reference [13] then introduces channel conditions in order to obtain expressions for the actual average error recovery time, and in certain cases, tighter upper bounds.

The purpose of this section is to extend the error recovery time results for the $N$ th-order nonlinear channel case. Our approach is based on the channel dependent results of [13]. Using the corresponding closed eye and open eye conditions based on the stability analysis results given in an earlier section, allows the selection of the appropriate error recovery time expressions. Secondly, the error recovery time expressions have been modified to account for the memory of the nonlinear
TABLE III

TABLE OF ERROR RECOVERY TIME EXPRESSIONS FOR $N$ TH-ORDER NONLINEAR CHANNELS

\begin{tabular}{|c|c|}
\hline Condition & Mean error recovery time \\
\hline $\begin{array}{l}\text { Any channel condition, any } \\
\text { SNR }\end{array}$ & $\begin{array}{l}M \leq T_{E R} \leq \frac{m\left((m)^{M}-1\right)}{(m-1)} \\
M=\max \left\{M_{1}, M_{2}, \ldots, M_{N}\right\}\end{array}$ \\
\hline Noise free, eye open & $\begin{array}{l}T_{E R}=M \\
=\max \left\{M_{1}, M_{2}, \ldots M_{N}\right\}\end{array}$ \\
\hline Noise free, eye closed & $\begin{array}{l}T_{E R}=\frac{m\left((m)^{M}-1\right)}{(m-1)} \\
M=\max \left\{M_{1}, M_{2}, \ldots M_{N}\right\}\end{array}$ \\
\hline $\begin{array}{l}\text { Noise free, last Volterra ker- } \\
\text { nels spanning a time period } \\
\text { of } r \text { samples give open eye } \\
\text { condition, while last Volterra } \\
\text { kernels spanning a time pe- } \\
\text { riod of } r+1 \text { samples strictly } \\
\text { close eye. }\end{array}$ & $\begin{array}{l}T_{E R}^{U}=\frac{m\left((m)^{M-r}-1\right)}{(m-1)}+r \\
M=\max \left\{M_{1}, M_{2}, \ldots, M_{N}\right\}\end{array}$ \\
\hline $\begin{array}{l}\text { Noise free, last Volterra ker- } \\
\text { nels spanning a time period } \\
\text { of } r \text { samples give open eye } \\
\text { condition, while last Volterra } \\
\text { kernels spanning a time pe- } \\
\text { riod of } r+1 \text { samples give non- } \\
\text { strict eye closure. }\end{array}$ & $\begin{array}{l}T_{E R}^{U}=\frac{2 m\left((m)^{M-r}-1\right)}{(m+1)(m-1)}+r \\
M=\max \left\{M_{1}, M_{2}, \ldots, M_{N}\right\}\end{array}$ \\
\hline
\end{tabular}

components. $T_{\mathrm{FR}}^{U}$ is the upper error recovery time bound, and $M=\max \left\{M_{1}, M_{2}, \ldots, M_{N}\right\}$. Error recovery bounds that apply for any channel condition, and any signal-to-noise ratio (SNR) are given. Average error recovery times based on channel (closed eye and open eye) conditions are given for the noiseless case, and generally apply for high SNR conditions. Tighter error recovery time bounds can be obtained by closely considering the eye condition during the error recovery time. The last Volterra kernels that span a time period $r+1$ samples may strictly close the eye [see (6)] or nonstrictly close the eye [see (7)]. But, the last Volterra kernels that span a time period of $r$ samples may not close the eye [see (8)]. In such a case, an initial, slow, closed eye error recovery will be followed by a fast, open eye error recovery for the last $r$ samples, allowing tighter error recovery time bounds to be determined. The results are summarized in Table III.

$$
\begin{aligned}
& 2(m-1) \sum_{i=M-r}^{M_{1}}\left|h_{i}\right|+2(m-1)^{2} \sum_{i=M-r}^{M_{2}} \sum_{j=i}^{M_{2}}\left|h_{i, j}\right|+\cdots \\
& +2(m-1)^{N} \sum_{i=M-r}^{M_{N}} \sum_{j=i}^{M_{N}} \cdots \sum_{q=p}^{M_{N}}\left|h_{i, j, \ldots, q}\right|>\left|h_{0}\right| \quad(6) \\
& 2(m-1) \sum_{i=M-r}^{M_{1}}\left|h_{i}\right|+2(m-1)^{2} \sum_{i=M-r}^{M_{2}} \sum_{j=i}^{M_{2}}\left|h_{i, j}\right|+\cdots \\
& +2(m-1)^{N} \sum_{i=M-r}^{M_{N}} \sum_{j=i}^{M_{N}} \cdots \sum_{q=p}^{M_{N}}\left|h_{i, j, \ldots, q}\right|=\left|h_{0}\right| \quad(7) \\
& 2(m-1) \sum_{i=M-r+1}^{M_{1}}\left|h_{i}\right|+2(m-1)^{2} \sum_{i=M-r+1}^{M_{2}} \sum_{j=i}^{M_{2}}\left|h_{i, j}\right|+\cdots \\
& +2(m-1)^{N} \sum_{i=M-r+1}^{M_{N}} \sum_{j=i}^{M_{N}} \cdots \sum_{q=p}^{M_{N}}\left|h_{i, j, \ldots, q}\right|<\left|h_{0}\right| .
\end{aligned}
$$




\section{CONCLUSION}

We have considered a DFE suitable for $N$ th-order nonlinear channels. We have shown the effect of the nonlinear terms by extending the stability criteria, error probability, and error recovery results to the $N$ th-order nonlinear channel case. The results presented are based on the error propagation over the maximum memory of the channel, including the memory of the nonlinear terms. The stability conditions take into account the nonlinear components and the maximum higher-order error values. The error probability and error recovery times take into account the memory of the nonlinear terms and the new stability conditions.

\section{REFERENCES}

[1] D. D. Falconer, "Adaptive equalization of channel nonlinearities in QAM data transmission systems," Bell Syst. Tech. J., vol. 57, no. 7, pp. 2589-2611, Sept. 1978.

[2] J. M. Cioffi, W. L. Abbott, H. K. Thapar, C. M. Melas, and K. D. Fisher, "Adaptive equalization in magnetic-disk storage channels," IEEE Commun. Mag., vol. 28, pp. 14-29, Feb. 1990.

[3] S. Chen, G. J. Gibson, C. F. N. Cowan, and P. M. Grant, "Adaptive equalization of finite nonlinear channels using multilayer perceptrons," Signal Processing, vol. 20, no. 2, pp. 107-119, June 1990.
[4] W. G. Jeon, J. S. Son, Y. S. Cho, Y. H. Lim, and D. H. Youn, "Nonlinear equalization for reduction of nonlinear distortion in high-density recording channels," in Proc. IEEE Int. Conf. Communications, Seattle, WA, June 1995, pp. 503-507.

[5] W. Frank and U. Appel, "Efficient equalization of nonlinear communication channels," in Proc. IEEE Int. Conf. Acoustics, Speech, and Signal Processing, Munich, Germany, Apr. 1997, pp. 2501-2504.

[6] V. J. Mathews, "Adaptive polynomial filters," IEEE Signal Processing Mag., vol. 8, pp. 10-26, July 1991.

[7] D. Duttweiler, J. E. Mazo, and D. G. Messerschmitt, "An upper bound on the error probability in decision-feedback equalization," IEEE Trans. Inform. Theory, vol. IT-20, pp. 490-497, July 1974.

[8] A. M. de Oliveira Duarte and J. J. O'Reilly, "Simplified technique for bounding error statistics for DFB receivers," Proc. IEEE, vol. 132-F, pp. 567-575, Dec. 1985

[9] R. A. Kennedy and B. D. O. Anderson, "Tight bounds on the error probabilities of decision feedback equalizers," IEEE Trans. Commun., vol. COM-35, pp. 1022-1028, Oct. 1987.

[10] S. A. Altekar and N. C. Beaulieu, "Upper bounds to the error probability of decision feedback equalization," IEEE Trans. Inform. Theory, vol. 39, pp. 145-156, Jan. 1993.

[11] A. Cantoni and P. Butler, "Stability of decision feedback inverses," IEEE Trans. Commun., vol. COM-24, pp. 970-977, Sept. 1976.

[12] R. A. Kennedy and B. D. O. Anderson, "Recovery times of decision feedback equalizers on noiseless channels," IEEE Trans. Commun., vol. COM-35, pp. 1012-1021, Oct. 1987.

[13] N. C. Beaulieu, "Bounds on recovery times of decision feedback equalizers," IEEE Trans. Commun., vol. 42, pp. 2786-2794, Oct. 1994.

[14] W. Feller, An Introduction to Probability Theory and its Applications, 2nd ed. New York: Wiley, 1957, vol. 1, ch. XIII, pp. 299-303. 